\title{
NifS-Mediated Assembly of [4Fe-4S] Clusters on NifU and Homologous Scaffold Proteins
}

Boi Hanh Huynh Emory University, United States

Guy N L Jameson, Department of Physics, Emory University, Atlanta, United States

Archer D Smith, IV, Department of Chemistry, University of Georgia, Athens, United States

Michael K Johnson, Department of Chemistry, University of Georgia, Athens, United States

Jeverson Frazzon, Department of Biochemistry, Virginia Tech, Blacksburg, United States

Dennis R Dean, Department of Biochemistry, Virginia Tech, Blacksburg, United States

Carsten Krebs, Department of Biochemistry and Molecular Biology, Pennsylvania, United States

Although Fe-S clusters are among the most ubiquitus and functionally diverse prosthetic groups in biology, biosynthesis of Fe-S clusters has only recently become the subject of extensive genetic, biophysical and biochemical investigations. These intensive activities followed the initial discovery that two nif gene products, NifS and NifU, of the nitrogen fixing bacterium Azotobacter vinelandii were essential for optimal assembly of $\mathrm{Fe}-\mathrm{S}$ clusters for the nitrogenase proteins, and the subsequent detection of homologous of nifS and nifU, termed iscS and iscU, respectively, that are part of a widely conserved prokaryotic operon involved with general Fe-S cluster biosynthesis (1). Both NifS and IscS catalyze the desulfuration of $\mathrm{L}$-cysteine to yield $\mathrm{L}$-alanine and elemental sulfur, thus providing the source of sulfur for Fe-S cluster assembly (1). NifU is a homodimeric protein containing a redox-active [2Fe-2S] cluster and additional site(s) that serve as a scaffold for the NifS-mediated assembly of Fe-S clusters (2). In this study, UV-Vis absorption and Mössbauer spectroscopies were used to investigate this complex cluster assembly process on NifU. The results show that each dimeric NifU can assemble up to two [4Fe-4S] clusters. Analysis of the time-dependent spectra suggests that oxidation of ferrous ion and reduction of the NifSbound cysteine persulfide are coupled to form an initial transient [2Fe-2S] cluster, followed by reductive coupling of the [2Fe-2S] clusters to form the [4Fe-4S] clusters. Comparison of results obtained for other scaffold proteins suggest that the coupled delivery of iron and sulfur by using ferrous ion to reduce sulfane sulfur, observed in NifU, is a common mechanism used by scaffold proteins. In addition, the ability to assemble [4Fe-4S] clusters was found to be a common attribute of all the NifU homologous investigated in our laboratories.

1 Zheng et al. (1998) J. Biol. Chem. 273, 13264, and references therein.

2 Yuvaniyama et al. (2000) Proc. Natl. Acad. Sci. USA 97, 599 